\title{
Cardiac Rehabilitation and Endurance Exercise Improves Heart Health Behaviors and Health Status: Do Patients Adhere?
}

\author{
Lydia Albuquerque*, Daria Napierkowski and Thomas Greey \\ Department of Nursing, College of Science and Health, William Paterson University, Wayne, New Jersey, USA
}

*Corresponding author: Lydia H Albuquerque, Assistant Professor, Department of Nursing, College of Science and Health, William Paterson University, Wayne, New Jersey, USA.
Received Date: November 27, 2019

Published Date: December 09, 2019

\begin{abstract}
World health organization has recognized Cardiac rehabilitation (CR) as a standard of care for prevention of cardiovascular disease such as cardiovascular mortality, cardiac death, stroke and non-fatal myocardial infarction. CR includes supervised training and self-care management recommended by the American Heart Association (AHA) and American College of Cardiology. Endurance type of exercise helps in improving the peak aerobic capacity (Peak V02).
\end{abstract}

Keywords: Cardiac rehabilitation; Heart health behaviors; Health status; Adherence; Endurance

\section{Introduction}

The leading cause of non-transmittable death in 2015 was cardiovascular disease which account for 17.9 million deaths worldwide [1]. Cardiac Rehabilitation (CR) is a clinically recommended and highly effective model for managing all forms of chronic cardiovascular diseases and its secondary symptoms [2]. Through the collection of empirical evidence, it has been shown that CR programs reduce total mortality by $13-24 \%$ in the one to three years following the initial coronary event [1]. The goal of CR is secondary prevention, which aims to detect and intervene with the progression of cardiovascular disease so as to decrease mortality and morbidity rates among patients. It is a cost-effective preventative program consisting of physical training, cardiac education, as well as counseling for cardiac health and risk [3]. It was recognized in 1993 by the World Health Organization as the standard of care for prevention of cardiovascular disease factors such as cardiovascular mortality, cardiac death, stroke, and non-fatal myocardial infarction [4]. CR exercise training has shown to improve clinical outcomes among cardiac patient population [5]. CR includes supervised training and self-care management recommended by the American Heart Association (AHA) and American College of Cardiology (ACC) [6]. Endurance type of exercise helps in improving the peak aerobic capacity (Peak VO2) of heart failure patients [7]. The HF- ACTION (Heart failure: A controlled Trial Investigating Outcomes of Exercise training) demonstrated a reduction in all-cause mortality rate and depression and improved quality of life [5]. Patient post diagnosis of cardiac disease attend CR at least twice a week for over 4-6 months. Many patients will require CR to improve Heart- Health Behaviors (HHB) and Health Status [2]. Patient adherence to prescribed CR is directly related to outcome [3].

\section{Phases of Cardiac Rehabilitation}

CR is broken down into several different phases. Phase I takes place during the acute period of time following the cardiac event. It focuses on cardiac education and rehabilitation of regular movement for functional independence by the time the patient is discharged from the hospital [8]. Phase II consists of structured and organized outpatient physical therapy and exercises on a regular weekly schedule directed towards heart health and prevention of secondary disease [8]. Phase III consists of group focused exercise and education directed towards forming lessons from the previous stages into lifestyle habits. Phase IV, the last phase, is the patient's own personal implementation of the exercises and information they have learned in the previous phases into their daily routines 
for the remainder of their lives [9]. The goal of all these phases, along with prevention of secondary symptoms, is to increase the health status of the patient [10] as well as their HHB [4].

\section{Heart health Behavior \& Health Status}

HHB consists of the activities in a person's life which effects the overall functioning of their heart. These are things such as whether or not a person smokes, keeps track of their blood pressure or hypertension, takes the necessary medications prescribed by their physician, has an unhealthy diet, or has sedentary life style. All these factors are a part of determining HHB [11]. Health Status is a multi-dimensional idea which is made up of many different indicators about the patient in question. One must take into account a person's physical, emotional, and social states to accurately determine health status. This means that factors such as if a patient is diagnosed with a long term disease or sickness, if their medical records show history of specific past issues, if the patient has an emotional or mental illness diagnosis should all be taken into account when determining Health status. Health status is also determined through the help of how the patient views their own health [12].

\section{Adherence to Cardiac Rehabilitation}

Adherence is the extent at which a patient's behavior corresponds with the suggested health recommendations from their physician. This can be anything from how often a patient remembers to take medication, to if they show up for their scheduled CR sessions, to whether or not a patient makes the recommended changes to their diet after release from the hospital [13]. Many different factors have been found responsible for the underuse and lack of adherence to CR programs. Factors such as old age and living at a distance greater than an hour from a rehabilitation center greatly impeded patients from participating in CR programs [10]. Patients with depression are seven times more likely to drop out of a CR program than those without depression. Patients who are single and have low social support have an increased risk factor of dropping out [1]. Lack of proper knowledge and perception of their disease [10] as well as being of a low socioeconomic status results in lower rates of adherence to programs [1]. Steps have been taken to try and address these factors and solve the issues which they present, such as suggesting free access to shuttle services for those who are a great distance from a CR center or are of low socioeconomic status. The implementation of providing heart risk education before $\mathrm{CR}$ begins for patients to understand the severity of the disease and increase attendance [1]. Another suggestion is for more in-home CR because those who did undertake in-home care showed greater rates of adherence to the program [3]. The suggestions to help increase adherence and attendance to CR programs are positive but they do not address the issue at present of knowing whether or not a patient's adherence to a program has a direct positive or negative effect on their heart health behavior and overall health status.

\section{Conclusion}

A study completed in Ontario, Canada, [14], determined that the sooner a patient is referred and admitted into a CR program after serious cardiac disease, the more likely a positive outcome and lower possibility of negative complication will occur in the following year. Along with this evidence, the 2016 European Guidelines on cardiovascular disease states that the more closely one adheres to a CR program's guidelines and treatment prescribed, the higher likelihood in preventing against secondary cardiovascular disease symptoms [15]. Despite the evidence that CR programs effectively reduce morbidity and mortality for cardiovascular disease patients, these programs are underutilized and lack patient adherence [1]. Based on the above review it is imperative to do further studies to elicit the effects of CR on HHB and health status of patients and explore the relationship of adherence to CR and its outcomes.

\section{Acknowledgement}

None.

\section{Conflict of Interest}

No conflict of interest.

\section{References}

1. Resurrección DM, Moreno-Peral P, Gómez-Herranz M, Rubio-Valera M, Pastor L, et al. (2019) Factors associated with non-participation in and dropout from cardiac rehabilitation programmes: A systematic review of prospective cohort studies. European Journal of Cardiovascular Nursing 18: 38-47.

2. Ali S, Chessex C, Bassett-Gunter R, Grace SL (2017) Patient satisfaction with cardiac rehabilitation: Association with utilization, functional capacity, and heart-health behaviors. Patient Preference and Adherence 11: 821-830.

3. Gostoli S, Roncuzzi R, Urbinati S, Morisky DE, Rafanelli C, et al. (2016) Unhealthy behaviour modification, psychological distress, and 1-year survival in cardiac rehabilitation. British Journal of Health Psychology 21(4): 894-916

4. Yancy CW, Jessup M, Bozkurt B, Butler J, Casey DE, et al. (2013) 2013 ACCF/AHA guideline for the management of heart failure: A report of the American College of Cardiology Foundation/American Heart Association task force on practice guidelines. Circulation 62(16): e147239.

5. Downing J, Balady GJ (2011) The role of exercise training in heart failure. J Am Coll Cardiology 58: 561-569.

6. Ades PA, Keteyian SJ, Balady GJ, Houston-Miller N, Kitzman DW, et al. (2013) Cardiac rehabilitation exercise and self-care for chronic heart failure. JACC Heart failure 1(6): 540-547

7. Grace SL, Midence L, Oh P, Brister S, Chessex C, et al. (2016) Cardiac rehabilitation program adherence and functional capacity among women: A Randomized Controlled Trial. Mayo Clinic Proceedings 91(2): 140-148.

8. Maddocks S, Cobbing S (2017) Patients experiences of and perspectives on phase 1 cardiac rehabilitation after coronary artery bypass graft surgery. Physiotherapy Canada 69(4): 333-340.

9. Del Pozo-Cruz B, Carrick-Ranson G, Reading S, Nolan P, Dalleck LC, et al. (2018) The relationship between exercise dose and health-related quality of life with a phase III cardiac rehabilitation program. Quality of Life Research 27(4): 993-998.

10. Endo N, Goto A, Suzuki T, Matsuda S, Yasumura S, et al. (2015) Factors associated with enrollment and adherence in outpatient cardiac 
rehabilitation in Japan. Journal of Cardiopulmonary Rehabilitation and Prevention 35(3): 186-192.

11. Nur KR (2018) Illness perception and cardiovascular health behavior among persons with ischemic heart disease in Indonesia. International Journal of Nursing Sciences 5(2): 174-180.

12. Madans JH, Webster KM (2015) Health Surveys. International Encyclopedia of the Social \& Behavioral Sciences (Second Edition): 725730 .

13. Jasmine TJ, Wai Chi SC, Hegney DG (2012) The impact of knowledge and beliefs on adherence to cardiac rehabilitation programs in patients with Heart Failure: A systematic review. JBI Library of Systematic Reviews 8(Supplement): 1-20.
14. Marzolini S, Blanchard C, Alter DA, Grace SL, Oh PI, et al. (2015) Delays in referral and enrolment are associated with mitigated benefits of cardiac rehabilitation after coronary artery bypass surgery. Circulation: Cardiovascular Quality and Outcomes 8(6): 608-620.

15. Hald K, Larsen FB, Nielsen KM, Meillier LK, Johansen MB, et al. (2019) Medication adherence, biological and lifestyle risk factors in patients with myocardial infarction: A ten-year follow-up on socially differentiated cardiac rehabilitation. Scandinavian Journal of Primary Health Care 37(2): 182-190. 